\title{
Controllable magnetic anisotropy and spin orientation of a prototypical easy-plane antiferromagnet on a ferromagnetic support
}

\author{
M. Ślęzak $\odot,{ }^{1,{ }^{*}}$ H. Nayyef $\odot,{ }^{1}$ P. Dróżdż, ${ }^{1}$ W. Janus, ${ }^{1}$ A. Kozioł-Rachwał, ${ }^{1}$ M. Szpytma, ${ }^{1}$ M. Zając, ${ }^{2}$ T. O. Menteş $\odot,{ }^{3}$ \\ F. Genuzio, ${ }^{4}$ A. Locatelli $\odot,^{3}$ and T. Ślęzak (iఠ ${ }^{1}$ \\ ${ }^{1}$ AGH University of Science and Technology, Faculty of Physics and Applied Computer Science, Kraków, Poland \\ ${ }^{2}$ National Synchrotron Radiation Centre SOLARIS, Jagiellonian University, Kraków, Poland \\ ${ }^{3}$ Elettra - Sincrotrone Trieste, Basovizza, Trieste, Italy \\ ${ }^{4}$ CERIC-ERIC, Basovizza, Trieste, Italy
}

(Received 2 September 2021; accepted 12 October 2021; published 28 October 2021)

\begin{abstract}
We report the magnetic properties of easy-plane antiferromagnetic $\mathrm{NiO}(111)$ thin films epitaxially grown on ferromagnetic $\mathrm{Fe}(110)$ layers. We find that the magnetic moments in $\mathrm{NiO}$ are rotatable within the $\mathrm{NiO}(111)$ sample plane and both magnetic anisotropy and orientation of antiferromagnetic NiO spins are determined by the magnetic properties of underlying ferromagnetic Fe layer. Specifically, the magnetic state and anisotropy of antiferromagnetic $\mathrm{NiO}$ can be modified by tuning the thickness of $\mathrm{Fe}$, changing the temperature, or applying small external magnetic field.
\end{abstract}

DOI: 10.1103/PhysRevB.104.134434

\section{INTRODUCTION}

During the past decades most of the spintronic applications relied on the manipulation of magnetic moments in ferromagnets (FM) [1], with antiferromagnets (AFM) [2] acting as passive elements only, for example as pinning layers in spin valves. Due to the large potential for downscaling and low power consumption, antiferromagnets are promising candidates for the next generation of spintronic devices [3-10]. Observation of skyrmions [11,12], spin Seebeck effect [13], or large anomalous Hall effect [14] in antiferromagnetic materials unlocks their already known as well as newly identified features for advantageous usage in spintronics. In this respect, the possibility to tailor the magnetic anisotropy [15] and control the spin orientation in AFM materials [16] is one of the emergent topics in solid-state physics. The rich variety of methods used to manipulate magnetic state of antiferromagnets can be classified in four general groups [17], i.e., optical, strain, electrical, or magnetic control. Methods based on magnetic interactions, which are at the focus of this paper, rely either on the application of strong external magnetic fields, which drive the transition to spin-flop state $[18,19]$, or on interfacial exchange coupling to a neighboring ferromagnet [20-22]. In the first case, the critical magnetic field required ranges from $\sim 1.6-9 \mathrm{~T}$ for $\mathrm{NiO}$ [19] up to even $70 \mathrm{~T}$ for $\mathrm{Mn}_{2} \mathrm{Au}[23,24]$, which is too large for applications and inconvenient for fundamental research. In the second case, the AFM-FM coupling at the interface can be employed to tune the magnetic properties of both ferromagnetic and antiferromagnetic components of the system. In AFM/FM systems the well-known exchange-bias effect $[25,26]$ is usually manifested by a shift of the hysteresis loop along the external magnetic field axis, as well as a coercivity enhancement [27] below blocking temperature. Depending on the

*Corresponding author: mislezak@agh.edu.pl coupling mechanism and the mutual influence of the spin arrangements between the AFM and FM components, a variety of interesting phenomena can be observed in both singleand polycrystalline materials as well as in core/shell nanoparticles [28]. Among these effects, magnetic domain patterns imprinting was demonstrated in both AFM [29] and FM [30] components of bilayered systems. Parallel and antiparallel coupling was discussed [31] together with the so-called positive exchange bias [31,32] and orthogonal coupling (similar to the classical "spin-flop" state in magnetically isolated antiferromagnets) was calculated [33] and experimentally observed [34]. In view of exchange-bias origin, its correlation with transition from rotatable to frozen antiferromagnetic spins in $\mathrm{CoO} / \mathrm{Fe}$ bilayers was studied [35]. Finally, the so-called rotatable magnetic anisotropy [36] was introduced in order to explain the coercivity enhancement in systems where the exchange-bias shift in the hysteresis loops was not detected.

In our approach we take the advantage of the exchange coupling strength at the interface of an epitaxial AFM/FM bilayer. The target of our research consists of the prototypical room-temperature antiferromagnet $\mathrm{NiO}$ [37,38], epitaxially grown on $\mathrm{Fe}(110) / \mathrm{W}(110)$, a model ferromagnetic system [39-41]. In bulk $\mathrm{NiO}$, below the Néel temperature $T_{\mathrm{N}} \approx$ $523 \mathrm{~K}$, the $\mathrm{Ni}^{2+}$ spins order ferromagnetically within $\{111\}$ planes with $\langle 11-2\rangle$ in-plane easy axes, while adjacent planes couple antiferromagnetically. This insulating antiferromagnet has recently regained huge attention as it can be used as a spin conductor or in $\mathrm{THz}$ frequency generators [42-44]. On the other hand, the magnetic properties of Fe thin films on W(110) single-crystal surface have been intensively studied for decades and are now well known [45-47]. As we will show, the advantages of $\mathrm{Fe}(110)$ films as supporting layer for $\mathrm{NiO}$ result from their structural and magnetic properties, which can be conveniently controlled by tuning film thickness, preparation conditions, or temperature [48]. The well-known Fe(110) spin reorientation transition (SRT) 
[40,49-51], which determines a rotation the Fe easy axis from [110] to the bulk-like [001] in-plane direction, proves to be especially advantageous for our purposes. Such [110] to [001] SRT can be driven either by increasing the Fe thickness [52] or decreasing the temperature [53] and is also observed in $\mathrm{Fe}(110)$ films coupled to an AFM layer, as we have recently reported for $\mathrm{CoO}(111) / \mathrm{Fe}(110)[54,55]$ and $\mathrm{NiO}(111) / \mathrm{Fe}(110)$ bilayers [56]. Here, we study the way in which the well-defined uniaxial magnetic anisotropy of ferromagnetic $\mathrm{Fe}(110)$ sublayer is directly imprinted onto the adjacent antiferromagnetic $\mathrm{NiO}(111)$ overlayer. Consequently, we also prove that the AFM magnetic state is fully determined by the current state of the FM component, and thus it can be controlled either thermally in a field-free manner or by means of very small external magnetic fields, orders of magnitude smaller than the reported spin-flop fields [19,23].

\section{EXPERIMENTAL DETAILS}

The Fe(110) films were grown by molecular-beam epitaxy on atomically clean W(110) single-crystal surfaces at room temperature and subsequently annealed at $675 \mathrm{~K}$ for $15 \mathrm{~min}$, prior to preparation of $\mathrm{NiO}$ overlayers. We made use of shutter shadowing the sample during Fe growth in order to prepare macroscopic regions with different Fe thicknesses $d_{\text {Fe }}$ covering the range $(50,150) \AA$. The sample prepared specifically for the $\mathrm{x}$-ray photoemission electron microscopy measurements (PEEM) presented in this paper consisted of two regions with 50- and $150-\AA$ Fe thickness. In order to document field-free, temperature-driven switching of $\mathrm{NiO}$ spins, also another sample consisting of several 300- $\mu \mathrm{m}$-wide $\mathrm{Fe}$ stripes, with the thickness in the range of (92-113 $\AA$ ), was prepared. In both samples, the $\mathrm{Fe}(110)$ surface was covered by $\mathrm{NiO}$ overlayers with homogeneous thickness $d_{\mathrm{NiO}}=40 \AA$. The $\mathrm{NiO}$ overlayers were grown at room temperature by reactive deposition of $\mathrm{Ni}$ in a partial oxygen pressure $P_{\mathrm{O} 2}=$ $1 \times 10^{-6}$ Torr. After each processing step, the structure of the surface was monitored in situ using low-energy electron diffraction (LEED).

The magnetic properties of the $\mathrm{NiO} / \mathrm{Fe}(110)$ bilayers were monitored by means of X-ray magnetic linear and circular dichroism (XMLD and XMCD). These analyses were complemented by both in situ and ex situ longitudinal magneto-optic Kerr effect (MOKE) measurements. Most of the spectroscopic data were obtained in the total electronyield (TEY) mode at the X-ray absorption spectroscopy (XAS) end-station of the Polish synchrotron SOLARIS [57]. The microscopy studies were carried out using the Spectroscopic PhotoEmission and Low Energy Electron Microscope (SPELEEM) at the Nanospectroscopy beamline of Elettra synchrotron (Trieste, Italy) [58]. In the SPELEEM setup the $\mathrm{x}$ rays are incident on the sample at $16^{\circ}$ grazing angle from the surface. Therefore, one of the two linear polarization states was within the sample plane, giving sensitivity to the change in the in-plane spin orientation of NiO. The XMLD-PEEM images were obtained by subtracting the two PEEM images (and normalizing to their sum) acquired at photon energies of 868.9 and $870.2 \mathrm{eV}$, which correspond to the two distinct absorption peaks within the Ni $L_{2}$ edge. The XMCD imaging with circular $\mathrm{x}$-ray polarization was mostly sensitive to inplane magnetization along the beam direction.

\section{RESULTS AND DISCUSSION}

The typical LEED pattern of Fe grown on the W(110) surface is shown in Fig. 1(a), where sharp diffraction spots clearly indicate a smooth unreconstructed (110) surface. Within the 50-200- $\AA$ thickness range of Fe, we find that the in-plane lattice spacing along the Fe[001] direction is $a_{001}=$ $2.88 \pm 0.02 \AA$. This matches well the value for bulk Fe and thus corresponds to an almost relaxed $\mathrm{Fe}(110) / \mathrm{W}$ film. The LEED pattern from the $\mathrm{NiO} / \mathrm{Fe}(110) / \mathrm{W}(110)$ surface, shown in Fig. 1(b), indicates a hexagonal $\mathrm{NiO}(111)$ surface structure independently from the thickness of the underlying Fe layer. Accordingly, ball models of $\mathrm{Fe}(110)$ and $\mathrm{NiO}(111) / \mathrm{Fe}(110)$ surfaces are presented in Figs. 1(c) and 1(d), respectively. From the LEED data analysis we can conclude that, within the surface plane, the $\mathrm{Fe}[001]$ and $\mathrm{Fe}[1 \overline{1} 0]$ in-plane directions are parallel to $\mathrm{NiO}[0 \overline{1} 1]$ and $\mathrm{NiO}[\overline{2} 11]$ directions, respectively. Additional LEED analysis was performed for $\mathrm{NiO} /(50 \AA$ $\mathrm{Fe})$ and $\mathrm{NiO} /(150 \AA \mathrm{Fe})$ regions of the sample, which was a subject of further magnetic characterization. From analysis presented in Supplemental Material [59] we conclude that both uncovered $\mathrm{Fe}(50 \AA)$ and $\mathrm{Fe}(150 \AA)$ as well as $\mathrm{NiO}(111) /(50 \AA \mathrm{Fe})$ and $\mathrm{NiO}(111) /(150 \AA \mathrm{Fe})$ regions can be treated as almost perfectly isostructural which means that all observed differences between these two regions have magnetic origin.

In Fig. 2(a) XAS spectra covering the $L_{3}$ and $L_{2}$ absorption edges of $\mathrm{Fe}$ are shown for $\mathrm{NiO}(111) /(50 \AA \mathrm{Fe})$ and $\mathrm{NiO}(111) /(150 \AA \mathrm{Fe})$ regions, as measured with left- and right-handed circular polarization of the beam. One can note that they significantly deviate from typical metallic Fe spectra, indicating partial oxidation of the topmost Fe layers, which results from reactive deposition of $\mathrm{NiO}$ overlayers. Please note that different preparation protocols were attempted in order to minimize this unwanted oxidation of Fe. Specifically, we tried to systematically increase the thickness of the initial metallic $\mathrm{Ni}$ layer grown before the oxygen pressure is increased in the preparation chamber. In such a way the oxidation of $\mathrm{Fe}$ can be avoided (if the reactive deposition of $\mathrm{NiO}$ starts above $\sim 4 \AA$ of metallic Ni) but this, on the other hand, leads to not fully oxidized $\mathrm{Ni}$ overlayer. We found a compromise between these two unwanted effects and consequently, all presented results are obtained for samples where 2 - $\AA$-thick metallic $\mathrm{Ni}$ is grown on the Fe surface before the reactive deposition of $\mathrm{NiO}$. For these samples the oxidation of $\mathrm{Fe}$ is minimized while the whole $\mathrm{Ni}$ layer is fully oxidized to $\mathrm{NiO}$, as confirmed by the lack of any detectable XMCD around $L_{3}$ and $L_{2}$ absorption edges of Ni (please see Supplemental Material [59]). A large $\mathrm{XMCD}$ asymmetry is observed in the thin Fe region, around $L_{3}$ and $L_{2}$ absorption edges of Fe, indicating that here the magnetization $\mathbf{M}$ is oriented along [110], which coincides with the X-ray propagation direction. On the contrary, there is no XMCD asymmetry on the thicker part of the sample, consistent with $\mathbf{M}$ being oriented along [001], perpendicular to the beam propagation vector (see Supplemental Material for corresponding differential XMCD spectra of Fe). This, together with MOKE data (not shown), directly confirms the 


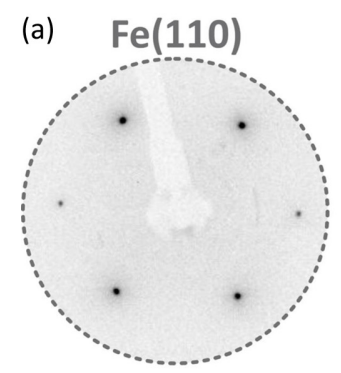

(c) $\bigcirc \mathrm{Fe}$

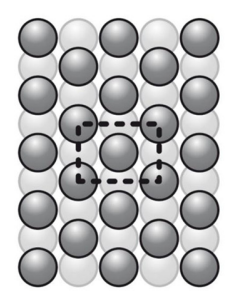

(b) $\mathrm{NiO}(111)$

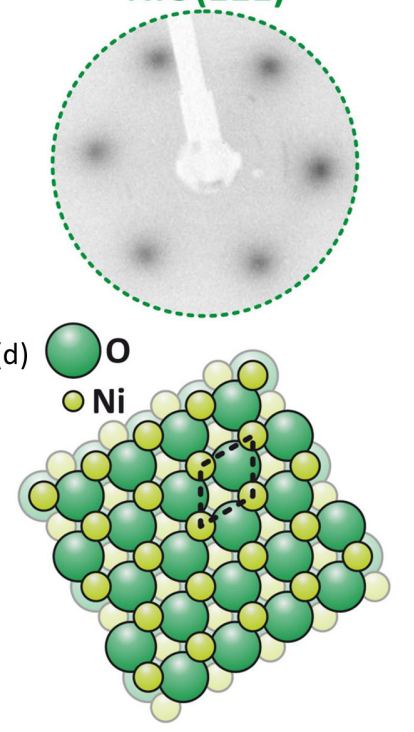

(e)

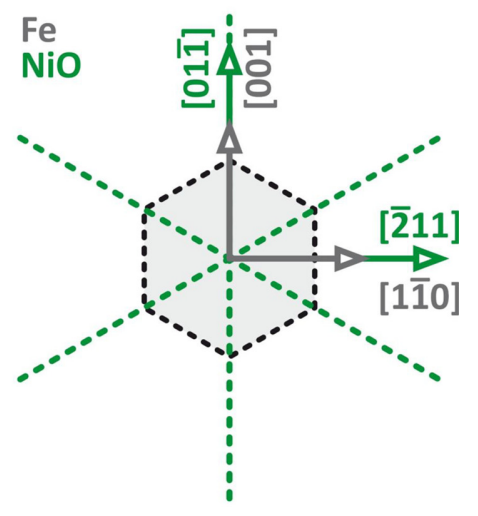

FIG. 1. LEED patterns of (a) uncovered $\mathrm{Fe}(110)$ and (b) $\mathrm{NiO}(111) / \mathrm{Fe}(110)$ surfaces. Corresponding ball models of (c) Fe(110) and (d) $\mathrm{NiO}(111) / \mathrm{Fe}(110)$ surfaces. The relative $\mathrm{Fe}$ and $\mathrm{NiO}$ in-plane directions as concluded from the LEED analysis are sketched in (e).

occurrence of thickness-induced SRT in $\mathrm{NiO} / \mathrm{Fe}(110)$ films. Figure 2(b) shows XAS spectra at the Ni $L_{2}$ edge from both sample regions, acquired with linear polarization of the photon beam parallel to the in-plane $\mathrm{Fe}[1 \overline{1} 0]$ direction. One can readily distinguish two peaks around the $L_{2}$ absorption edge of Ni. The ratio of these two intensity maxima, called $R_{L 2}$, is routinely used as a probe of $\mathrm{NiO}$ magnetic moments orientation $[60,61]$. The small but noticeable XMLD effect, visible as a difference between two XAS spectra in Fig. 2(b), indicates that the thickness-induced $\mathrm{Fe}[1 \overline{1} 0]$ to $\mathrm{Fe}[001] \mathrm{SRT}$ in $\mathrm{Fe}$ is accompanied by a $\mathrm{NiO}[\overline{2} 11] \| \mathrm{Fe}[1 \overline{1} 0]$ to $\mathrm{NiO}[01 \overline{1}] \| \mathrm{Fe}[001]$ SRT in antiferromagnetic NiO. This result is strongly supported by the XMCD- and XMLD-PEEM data presented in Fig. 2(c), top and center panel, respectively. Clearly, in the vicinity of 50/150- $\AA \mathrm{Fe}$ border, the magnetic domain pattern of $\mathrm{Fe}$, visible in XCMD-PEEM image, is directly reflected in the $\mathrm{NiO}$ magnetic structure, as seen in the corresponding XMLD-PEEM image acquired with polarization parallel to the sample surface plane [Fig. 2(c), middle]. In the lower panel of Fig. 3(c), an XMLD-PEEM image acquired with
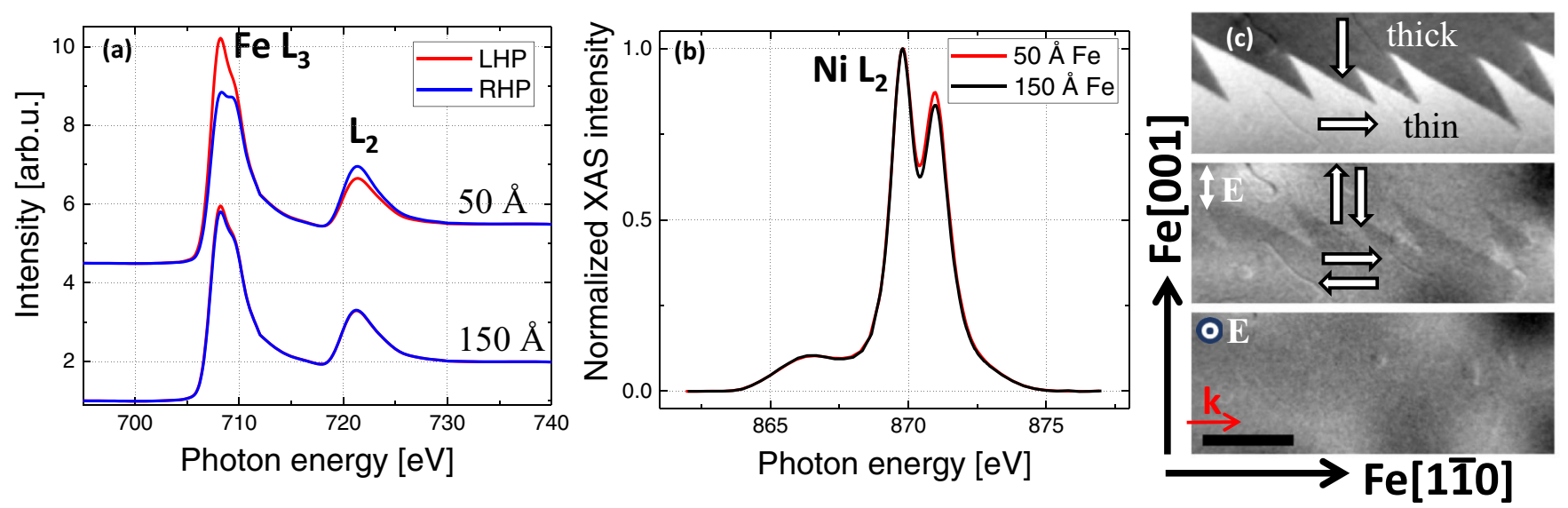

FIG. 2. (a) Fe $L_{2,3}$ XMCD spectra from the $\mathrm{NiO}(111) /(50 \AA \mathrm{Fe})$ and $\mathrm{NiO}(111) /(150 \AA \mathrm{Fe})$ regions of the sample. LHP and RHP stand for circular left- and right-handed polarization, respectively. (b) Ni $L_{2}$ XMLD spectra acquired on two sample regions using x rays with linear polarization vector parallel to the in-plane $\mathrm{Fe}\left[1 \overline{1} 0\right.$ ] direction. (c, top panel) $\mathrm{Fe} L_{3}$ XMCD-PEEM image of the $\mathrm{SRT}$ in $\mathrm{NiO} / \mathrm{Fe}(110)$ at the $150-\AA \mathrm{Fe}$ (up)/50- $\AA \mathrm{Fe}$ (down) boundary. The image contrast originates from the relative alignment of the photon propagation direction $\mathbf{k}$ and magnetization M, (c, center panel) corresponding Ni $L_{2}$ XMLD-PEEM image of the same area obtained with x-ray polarization along in-plane $\mathrm{Fe}$ [001] direction. In the bottom panel of (c) XMLD image obtained with x-ray polarization along the surface normal is shown. Modulations of the image intensity, appearing as intensity minima and maxima, affect the XMLD images, superimposed to the magnetic signal. These artifacts are caused by the inhomogeneity of the (improperly) focused x-ray-beam spot on the sample, which drifts during image acquisition. Scale bar in (c) corresponds to $5 \mu \mathrm{m}$. Intensity profiles across the domain boundary for both XCMD and XMLD images are presented in Supplemental Material [59]. 
(a)

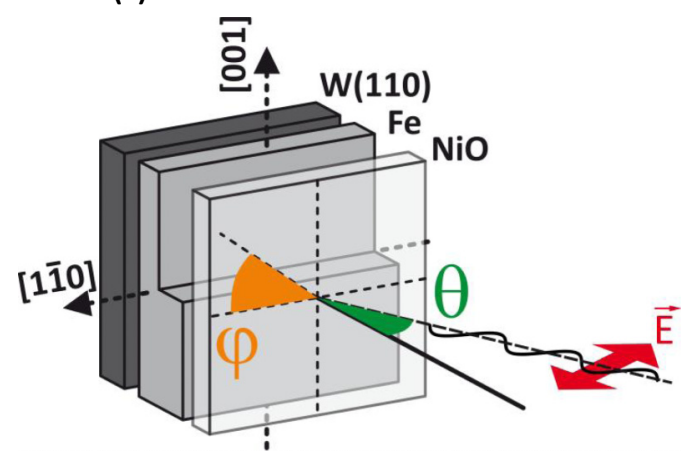

(b)

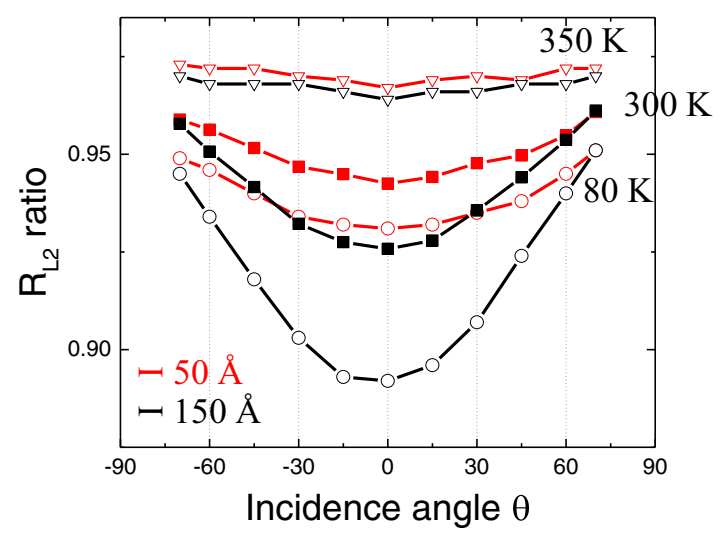

(c)
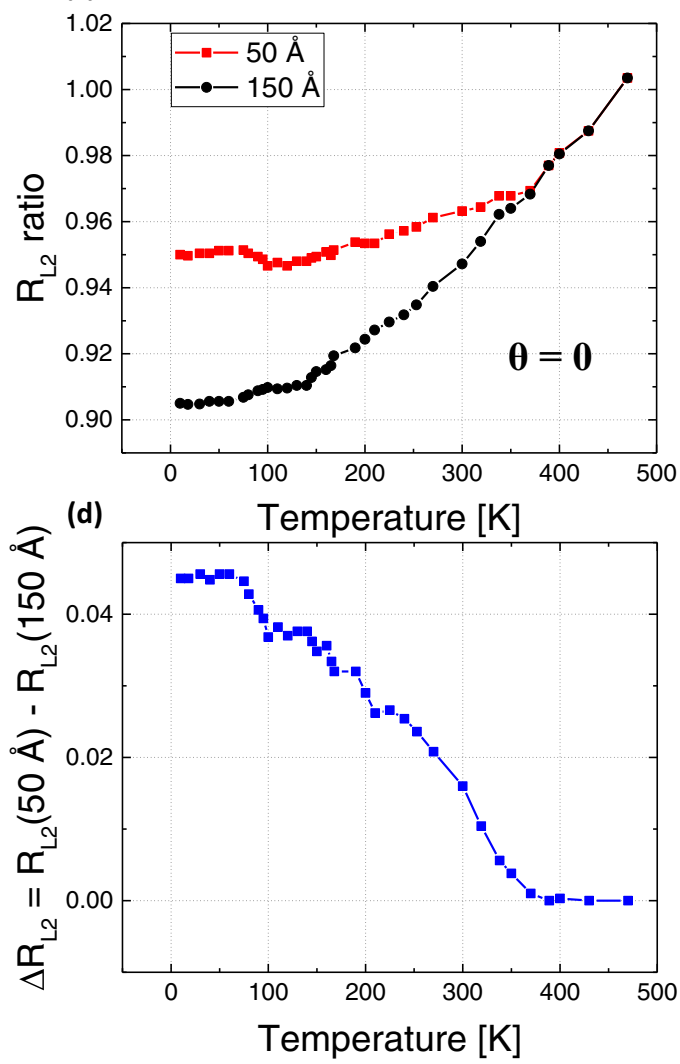

FIG. 3. (a) Schematic sketch of the angle-resolved XMLD measurements geometry. (b) $R_{L 2}$ ratio dependence on the polar angle $\theta$ (fixed $\varphi=0$ ) for $80 \mathrm{~K}$ (circles), $300 \mathrm{~K}$ (squares), and $350 \mathrm{~K}$ (triangles), for both studied Fe thicknesses, $50 \AA$ (red) and $150 \AA$ (black). (c) Temperature dependence of $R_{L 2}$ ratio for $\mathrm{NiO} /(50 \AA \mathrm{Fe})$ and $\mathrm{NiO} /(150 \AA \mathrm{Fe})$ regions of the sample. (d) Temperature dependence of difference of $R_{L 2}$ ratios from (c) defined as $\Delta R_{L 2(\theta=0)}=R_{L 2}(50 \AA)-R_{L 2}(150 \AA)$.

linear polarization, which is close to the surface normal, is shown. In this case the lack of magnetic contrast confirms the conclusion that $\mathrm{NiO}$ spins are confined within $\mathrm{NiO}(111)$ sample plane, as expected. We note that XMCD-PEEM images acquired at the $\mathrm{Ni} L_{3}$ edge (not shown) do not exhibit any magnetic contrast across the 50/150- $\AA$ Fe border, confirming that the contrast visible in XMLD-PEEM image in Fig. 2(c) originates purely from the antiferromagnetic ordering in NiO. Thus, Fig. 2 proves that in-plane SRT is observed across the 50/150- $\AA$ Fe border in both ferromagnetic Fe and antiferromagnetic $\mathrm{NiO}$ sublayers of $\mathrm{NiO}(111) / \mathrm{Fe}(110)$ bilayer.

In order to follow the magnetic properties of antiferromagnetic $\mathrm{NiO}(111)$ overlayers in detail, systematic angle-resolved and temperature-dependent XMLD measurements were performed, which are depicted in Fig. 3(a) along with the sketch of experimental geometry. First, the $R_{L 2}$ ratio dependence on the polar angle $\theta$ was followed for various temperatures [Fig. 3(b)] and fixed azimuthal angle $\varphi=0$. In such geometry the electric field vector $\mathbf{E}$ projection on the $\mathrm{NiO}(111) \| \mathrm{Fe}(110)$ sample plane is parallel to the $\mathrm{NiO}[\overline{2} 11] \| \mathrm{Fe}[1 \overline{1} 0]$ in-plane direction. Consequently, on 50-Å-thick Fe region increasing the polar $\theta$ angle is equivalent to increasing the angle between $\mathbf{E}$ and $\mathrm{NiO}$ spins. On the contrary, in the 150- $\AA$-thick Fe region, the angle between $\mathbf{E}$ and $\mathrm{NiO}$ spins stays constant as the $\theta$ angle is changing. Naively, one would expect strong dependence of $R_{L 2}(\theta)$ on the 50 - $\AA$-thick Fe region and no dependence on 150 - $\AA$-thick Fe region. This is obviously not the case in Fig. 3(b), where $R_{L 2}$ ratio at $80 \mathrm{~K}$ strongly depends on $\theta$ for $150-\AA$ region (black circles) while on 50 - $\AA$-thick $\mathrm{Fe}$ region (red circles) the corresponding dependence is much weaker. This seemingly surprising observation is consistent with a previous report [48], resulting from the fact that the XMLD asymmetry in $\mathrm{NiO}$ depends not only on the relative orientation of electric field $\mathbf{E}$ and AFM spins, but also the angular dependence with respect to the crystallographic axes has to be taken into account $[60,62]$. Low-temperature angular dependencies of $R_{L 2}$ ratios (see Fig. 3) confirm the in-plane confinement of $\mathrm{NiO}$ spins and their collinear coupling to adjacent $\mathrm{Fe}$ magnetic moments, as expected for uncompensated $\mathrm{NiO}(111)$ interface and in agreement with previous report on $\mathrm{NiO}(111) / \mathrm{CoFe}$ bilayers [63]. With increasing temperature, the observed angular dependence is continuously damped and at $350 \mathrm{~K}$ the $R_{L 2}$ ratio is almost independent of $\theta$ for both sample regions. This means that the Néel temperature of 40-Â-thick $\mathrm{NiO}(111)$ is significantly reduced as compared to its bulk value due to finite-size effects, in agreement with the pioneering work on (001)-oriented NiO films [64].

Systematic, temperature-dependent XAS measurements were performed in normal incidence geometry $(\theta=0)$ with $\mathbf{E}\|\mathrm{NiO}[\overline{2} 11]\| \mathrm{Fe}[1 \overline{1} 0](\varphi=0)$. The corresponding results are shown in Fig. 3(c) $\left(R_{L 2}\right.$ ratios for both sample regions) and in Fig. 3(d) where the difference of two $R_{L 2}$ ratios defined as 


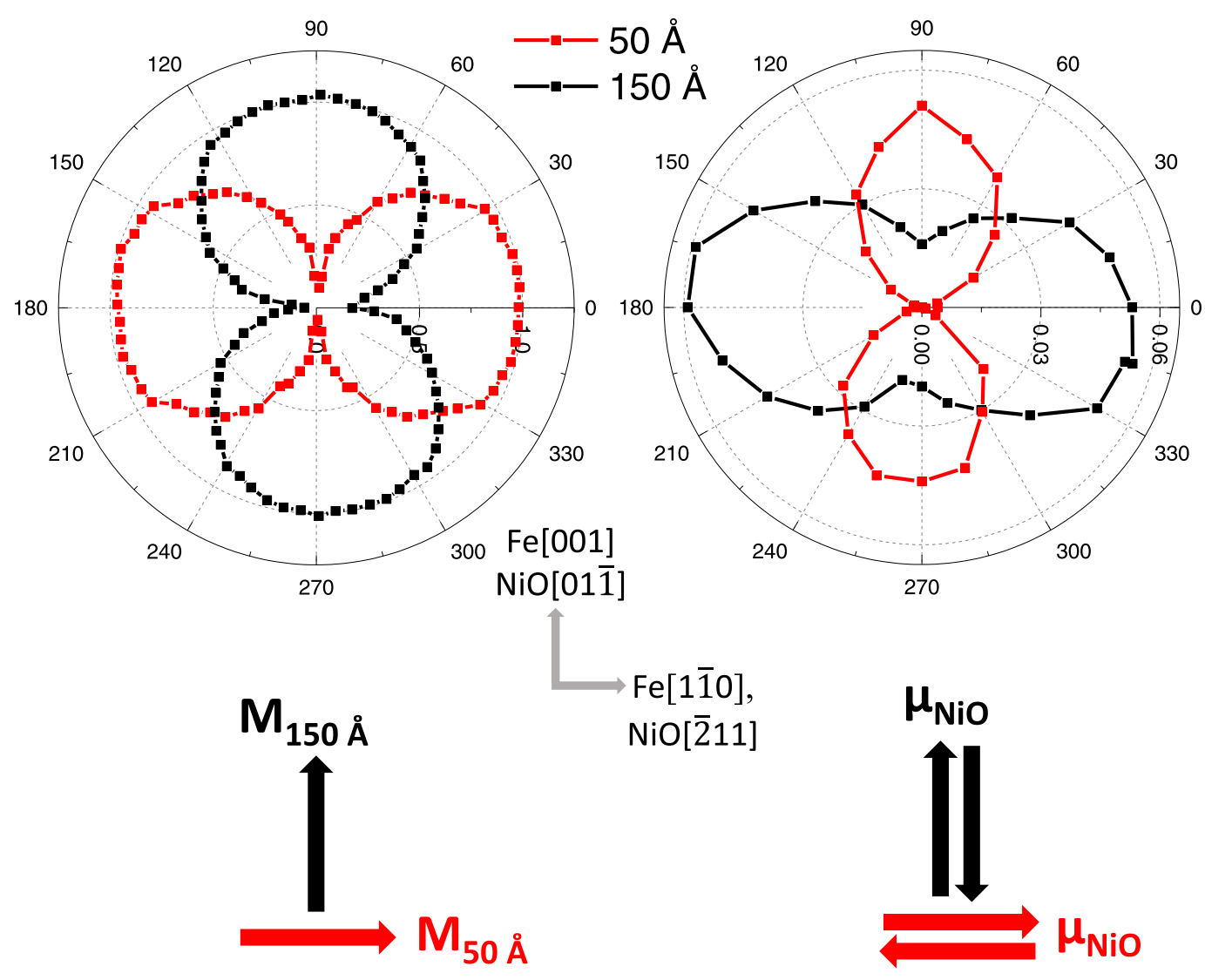

FIG. 4. In-plane angular dependencies of (left) Fe magnetization in remanent state as determined from MOKE hysteresis loops and (right) XMLD magnitude defined as $\Delta R_{L 2 \varphi}=\left|R_{L 2}\left(\theta=0^{\circ}\right)-R_{L 2}\left(\theta=60^{\circ}\right)\right|$, as a function of the in-plane angle $\varphi$. Arrows at the bottom of the image schematically mark the easy axes of ferromagnetic Fe and antiferromagnetic NiO sublayers for both sample regions. Red and black colors correspond to 50- and 150- $\AA$ sample regions.

$\Delta R_{L 2(\theta=0)}=R_{L 2}(50 \AA)-R_{L 2}(150 \AA)$ can be seen. Based on these results, the Néel temperature of $40-\AA \AA$-thick $\mathrm{NiO}(111)$ layer can be roughly estimated to be $\sim 380 \mathrm{~K}$. This value is consistent with the reported $T_{\mathrm{N}}=295,430$, and $470 \mathrm{~K}$ found for the 5, 10, and 20 monolayers of (001)-oriented $\mathrm{NiO}$ films [64]. As discussed in Ref. [64], in order to prepare NiO layer with bulk Néel temperature, one would need to exceed at least a thickness of 30 monolayers, i.e., $\approx 60 \AA$. For such thick $\mathrm{NiO}$ layers, XAS measurements performed in TEY mode would not provide enough sensitivity to the buried $\mathrm{NiO} / \mathrm{Fe}$ interface, due to the limited probing depth, which for $\mathrm{NiO}$ is estimated to be around $50 \AA$. Additionally, one can note the variation of $R_{L 2}$ ratio above Néel temperature which can be attributed to the spin-spin correlation effects that influence the shape of the isotropic XAS spectrum as shown in Ref. [64].

We now focus on the in-plane magnetic anisotropy of both $\mathrm{Fe}$ and $\mathrm{NiO}$ sublayers. The corresponding results are shown in Fig. 4, left and right panels, respectively. In case of ferromagnetic $\mathrm{Fe}(110)$ sublayer its in-plane magnetic anisotropy was followed by angle-dependent MOKE measurements. From magnetic hysteresis loops the magnetization in the remanence state was determined and plotted as a function of in-plane azimuthal angle $\varphi$ (Fig. 4, left panel). The presented results clearly confirm the existence of strong uniaxial in-plane magnetic anisotropy, which, for 50 - $\AA$-thick Fe, favors the $\mathrm{Fe}[1 \overline{1} 0]$ easy axis, while for $150-\AA$-thick Fe favors the bulk- like Fe[001] orientation. Such twofold symmetry of in-plane magnetic anisotropy is mimicked by neighboring antiferromagnetic $\mathrm{NiO}$. Its magnetic anisotropy was followed by measuring XAS spectra as a function of $\varphi$ angle. For each given $\varphi$ angle, two spectra were collected, one for $\theta=0$ (normal incidence geometry) and second for $\theta=60^{\circ}$ (close to grazing incidence geometry). $R_{L 2}$ ratios were determined for both geometries and their difference is defined as $\Delta R_{L 2 \varphi}=$ $\left|R_{L 2}\left(\theta=0^{\circ}\right)-R_{L 2}\left(\theta=60^{\circ}\right)\right|$. In the right panel of Fig. 4, corresponding plots of $\Delta R_{L 2 \varphi}(\varphi)$ as a function of the in-plane angle $\varphi$ are shown for both thicknesses of Fe sublayer. Instead of threefold in-plane symmetry observed for bulk $\mathrm{NiO}(111)$ in early works [65], exchange coupling with neighboring $\mathrm{Fe}$ layer makes the in-plane magnetic anisotropy of NiO twofold with its easy axis determined by the magnetic anisotropy of the ferromagnet. Specifically, due to collinear coupling between $\mathrm{NiO}$ and $\mathrm{Fe}$ spins at the interface, the easy axis of $\mathrm{NiO}$ is parallel to $\mathrm{NiO}[\overline{2} 11] \| \mathrm{Fe}[1 \overline{1} 0]$ and $\mathrm{NiO}[0 \overline{1} 1] \| \mathrm{Fe}[001]$ on 50- and 150-Å-thick regions, respectively. One can note that while for $\mathrm{NiO} /(50 \AA \mathrm{Fe})$ region the XMLD effect totally vanishes along the $\mathrm{Fe}$ easy axis $(\varphi=0)$, its value is nonzero $(\sim 25 \%$ of maximum XMLD) for $\mathrm{NiO} /(150 \AA \mathrm{Fe})$ sample area along Fe easy axis at $\varphi=90^{\circ}$. This suggests that on thick Fe films some additional magnetic anisotropy of $\mathrm{NiO}$ is showing up. This can be understood bearing in mind conclusions from LEED results presented in Fig. 1. The easy axis of thin Fe, 


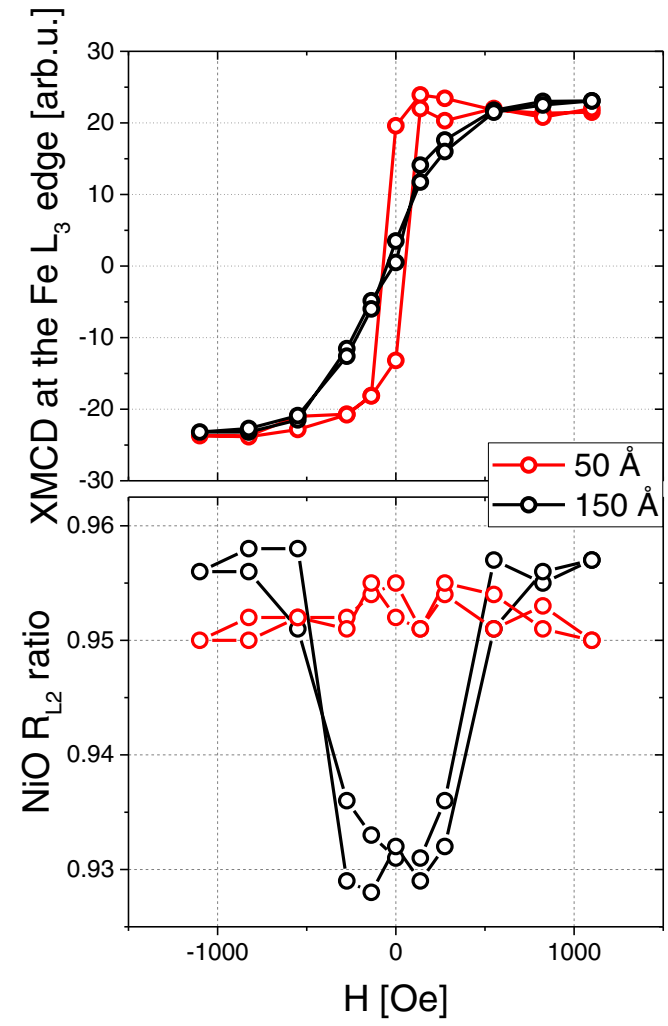

FIG. 5. Element-sensitive XMCD (top) and XMLD (bottom) magnetic hysteresis loops of $\mathrm{Fe}$ and $\mathrm{NiO}$ sublayers, respectively.

$\mathrm{Fe}[1 \overline{1} 0]$, is aligned with one of three easy axes of bulk NiO, namely with $\mathrm{NiO}[\overline{2} 11]$ direction. Consequently, both interaction with $\mathrm{Fe}$ and intrinsic $\mathrm{NiO}$ magnetic anisotropy make the $\mathrm{NiO}[\overline{2} 11] \| \mathrm{Fe}[1 \overline{1} 0]$ orientation of AFM spins favorable. This is not the case on $\mathrm{NiO} /(150 \AA \mathrm{Fe})$ sample region. The easy axis of thick $\mathrm{Fe}, \mathrm{Fe}[001]$, does not coincide with any of the easy axes of bulk $\mathrm{NiO}$ directions $\langle 211\rangle$ but it lies exactly between two of them, i.e., $30^{\circ}$ away from both. As a result, the intrinsic contribution to the effective magnetic anisotropy of $\mathrm{NiO}$ influences the angle-dependent XMLD results and causes its nonzero value for $\varphi=90^{\circ}$.

Beyond the issue of in-plane magnetic anisotropy of both FM and AFM components of $\mathrm{NiO} / \mathrm{Fe}$ bilayer there is a question concerning $\mathrm{NiO}$ spin orientation during the magnetization reversal of Fe. Specifically, in case of the so-called rotatable antiferromagnetic spins [35], their orientation can be changed for example by application of external magnetic fields. In the second scenario, namely frozen AFM spins [35], their orientation is insensitive to any switching of FM magnetization induced by external fields, temperature, etc. In Fig. 5 we show element-sensitive magnetic hysteresis loops measured by means of XMCD and XMLD for ferromagnetic Fe and antiferromagnetic NiO sublayers, respectively. During measurements external magnetic field was applied in the geometry in which $\left(\varphi=0, \theta=60^{\circ}\right)$, and thus the in-plane component of magnetic field was along $\mathrm{Fe}[1 \overline{1} 0]$ direction. Please note that due to large in-plane magnetic anisotropy of $\mathrm{Fe} / \mathrm{W}(110)$ films, the nonzero out-of-plane component of external magnetic field has no effect on Fe magnetic state as orders of

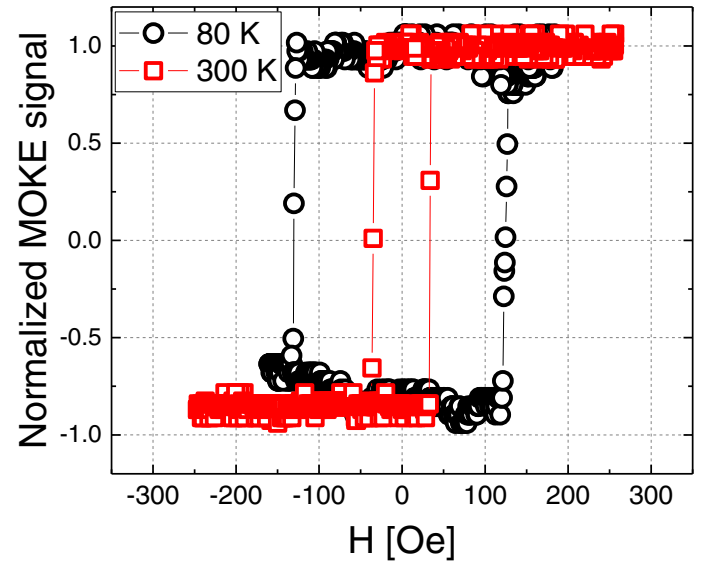

FIG. 6. Easy-axis magnetic hysteresis curves for $\mathrm{NiO} /(50 \AA \mathrm{Fe})$ region as measured by MOKE at 300 and $80 \mathrm{~K}$; external magnetic field was applied along $\mathrm{Fe}[1 \overline{1} 0]$ in-plane direction.

magnitude higher fields are necessary to switch $\mathrm{Fe}(110)$ magnetic moments out of (110) sample plane. A typical square hysteresis curve is observed for the thin $\mathrm{Fe}$ region, which is below the SRT critical thickness, while a hard axis loop with almost zero magnetization in remanent state is seen for the $150-\AA$-thick Fe. In case of $\mathrm{NiO}$, on the $50-\AA$-thin $\mathrm{Fe}$ region, external magnetic field makes no changes in XMLD as it is insensitive to a $180^{\circ}$ reversal. On the contrary, on the thick Fe region one can clearly see a change of $\mathrm{Ni} R_{L 2}$ as a function of external magnetic field. This observation can be easily understood by considering magnetization reversal of 150- $\AA$-thick $\mathrm{Fe}$ along its hard magnetic anisotropy axis and interfacial exchange coupling between $\mathrm{Fe}$ and $\mathrm{NiO}$ spins. Above the in-plane anisotropy field of 150 - $\AA$-thick Fe sublayer $(\sim 750$ Oe) the magnetization is forced to align along the hard axis, i.e., $\mathrm{Fe}[1 \overline{1} 0]$. As the field is reduced, the Fe magnetic moments reorient by $90^{\circ}$ and align with their easy axis, i.e., $\mathrm{Fe}[001]$. Due to exchange coupling at the $\mathrm{NiO} / \mathrm{Fe}$ interface such reorientation is also accompanied by in-plane $90^{\circ}$ switching of $\mathrm{AFM} \mathrm{NiO}$ spins.

The data shown in Fig. 5 prove clearly that $\mathrm{NiO}$ spins in $\mathrm{NiO} / \mathrm{Fe}$ bilayer are rotatable and their orientation can be easily changed, for example by applying small external magnetic field on the order of 500 Oe. Observation of rotatable AFM spins agrees well with the fact that no exchange bias (no shift field along the external magnetic field axis) is observed in our $\mathrm{NiO}(111) / \mathrm{Fe}(110)$ system, as can be seen from magnetic hysteresis loops of Fe (Fig. 5, top) that are symmetric with respect to $H=0$ axis. The same observation was also confirmed by numerous magnetic hysteresis loops acquired for $\mathrm{NiO}(111) / \mathrm{Fe}(110)$ bilayers with MOKE, as for example the one measured at $80 \mathrm{~K}$ for $\mathrm{NiO} /(50 \AA \mathrm{Fe})$ with external magnetic field applied along Fe[1/10] direction; see Fig. 6 (black loop). It has to be also noted that applying a fieldcooling procedure with starting temperature well above $\mathrm{NiO}$ Néel temperature does not lead to the onset of exchange bias and does not freeze the AFM spins of $\mathrm{NiO}$, in contrast with isostructural $\mathrm{CoO}(111) / \mathrm{Fe}(110)$ system where large exchange bias can be easily induced and frozen antiferromagnetic spins of $\mathrm{CoO}$ observed [54]. This means that $\mathrm{NiO}$ spins are fully 


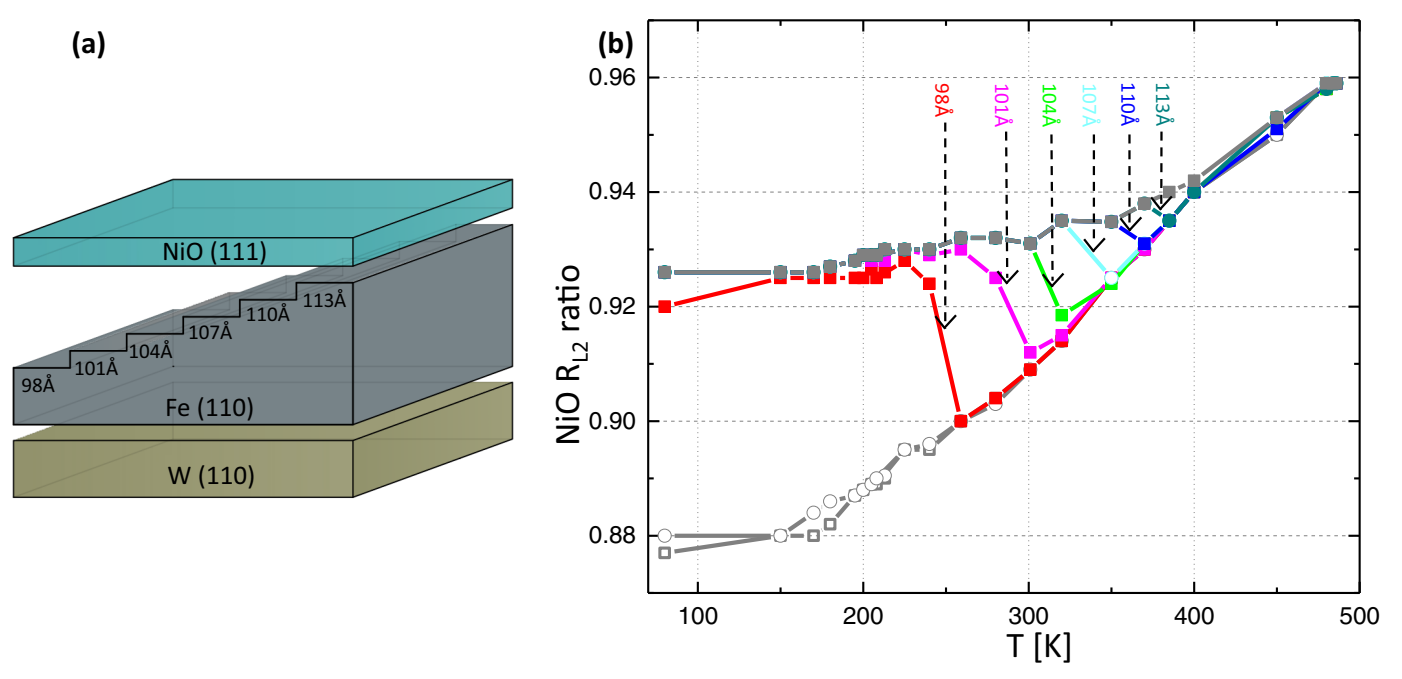

FIG. 7. (a) Schematic sketch of the sample specially prepared in order to present tuning of SRT critical temperature in NiO/Fe bilayer. (b) Temperature dependence of XMLD $\left(R_{L 2}\right)$ shows SRT in AFM NiO during heating process for various Fe(110) thicknesses. Results were obtained in normal incidence geometry $\theta=0^{\circ}$ and with azimuthal angle $\varphi=90^{\circ}$ which means $\mathbf{E} \| \mathrm{Fe}[001]$.

rotatable at low temperature, which can be attributed to much smaller intrinsic magnetic anisotropy of $\mathrm{NiO}$ as compared to magnetically hard $\mathrm{CoO}$. The absence of the shift of the hysteresis loops could potentially be also explained by possible spin-flop coupling between $\mathrm{Fe}$ and $\mathrm{NiO}$ magnetic moments. Such orthogonal coupling has been however already excluded by angle-dependent XMLD studies presented in Figs. 3 and 4. Also, this conclusion corresponds well to what is expected for nominally fully uncompensated $\mathrm{NiO}(111)$ interface. It has to be however underlined that the AFM-FM coupling is also manifested in the coercivity enhancement. In Fig. 6 the easyaxis hysteresis curves for $\mathrm{NiO} /(50 \AA \mathrm{Fe})$ region are presented, as measured by MOKE at 300 and $80 \mathrm{~K}$. A significant increase of the coercive field is observed with decreasing temperature, which can be interpreted in terms of rotatable anisotropy [66] that apparently turns on below Néel temperature of $\mathrm{NiO}$ overlayer. Note that such temperature-induced dependence of coercivity can only be attributed to FM-AFM coupling as for the AFM-free systems the coercive field of $\mathrm{Fe}(110)$ films very weakly depends on the temperature. For example the coercive field of 60-Å-thick $\mathrm{Fe}(110)$ film in $\mathrm{W}(110) / \mathrm{Fe}(110) / \mathrm{W}(110)$ epitaxial stack changes from $\sim 30$ to $\sim 40$ Oe as the temperature decreases from 300 to $10 \mathrm{~K}$ [67]. In contrast, in our $\mathrm{NiO} / \mathrm{Fe}(110)$ system, due to AFM-FM interaction the coercive field increases from $\sim 30$ Oe at $300 \mathrm{~K}$ to $\sim 130 \mathrm{Oe}$ at $80 \mathrm{~K}$. This means that the detectable rotatable anisotropy is present in our AFM/FM system, although its magnitude is much smaller as compared for example to Ref. [36]. This again can be understood bearing in mind intrinsically small and nonuniaxial magnetic anisotropy of $\mathrm{NiO}$, in contrast to magnetically much "harder" antiferromagnet $\mathrm{CoO}$ [36].

Thus, the rotatable nature of $\mathrm{NiO}$ spins suggests that their switching can be induced in a field-free manner, for example by combining AFM-FM exchange coupling with the temperature-induced SRT in the FM sublayer. Recently we showed that in a uniform thickness $\mathrm{NiO}(111) / \mathrm{Fe}(110)$ epitaxial bilayer system, two magnetic states with orthogonal spin orientations can be stabilized at a given temperature near $250 \mathrm{~K}$ in antiferromagnetic $\mathrm{NiO}$ due to thermal hysteresis of temperature-induced SRT in both FM and AFM sublayers [56]. As a result, field-free, reversible switching between these two AFM states was demonstrated. In order to make a systematic investigation of such field-free, temperature-induced switching of AFM NiO spins, a sample which contained several 300- $\mu \mathrm{m}$-wide Fe stripes [Fig. 7(a)] with different thicknesses in the range of (92-113 $\AA$ ), was prepared. Figure 7(b) shows the temperature dependence of the $R_{L 2}$ ratio for various thicknesses of Fe. As the thermal expansion of the sample manipulator can reach up to millimeter scale for the temperature range from 80 to $300 \mathrm{~K}$, one has to stabilize a given temperature for a long time in order to be able to correct for drifts and to provide reliable measurements on particular stripes with well-defined Fe thickness. For such procedure in case of systematic temperature-dependent measurements at a synchrotron facility, time constraints become important. For this reason, only the heating branch of the temperature-induced SRT is shown in Fig. 7(b), while cooling branches were not monitored for all Fe stripes. Instead, we refer to the Supplemental Material [59], in which full coolingheating thermal hysteresis of both XMCD and XMLD (for two experimental geometries) are shown, as determined for a homogeneous sample with selected uniform Fe thickness. From Fig. 7(b) it is clear that depending on the Fe thickness a critical temperature of SRT in AFM NiO can be tuned precisely and in a broad range. With increasing Fe thickness the critical temperature, at which $\mathrm{NiO}$ spins rotate in plane by $90^{\circ}$ towards the $\mathrm{Fe}[1-10]$ direction, continuously increases. This provides the possibility to cover a wide temperature window $\sim(250-370 \mathrm{~K})$ for field-free, reversible switching of AFM spins.

\section{CONCLUSIONS}

The magnetic properties of prototypical, easy-plane antiferromagnetic $\mathrm{NiO}(111)$ films supported by ferromagnetic $\mathrm{Fe}(110)$ layers were characterized by means of spectroscopic and microscopic XMLD techniques. $90^{\circ}$ spin reorientation transition can be induced in the easy planes of both $\mathrm{NiO}(111)$ 
and $\mathrm{Fe}(110)$ sublayers of AFM/FM bilayers, either by increasing the $\mathrm{Fe}$ thickness or by changing the temperature. The latter case allowed us to implement field-free switching of antiferromagnetic spins between two orthogonal in-plane orientations. The critical temperature of such AFM reversal can be precisely tuned in a range from 250 to $370 \mathrm{~K}$, which, importantly, covers room temperature. Element-sensitive magnetic hysteresis loops collected for FM and AFM components of the bilayer system prove that $\mathrm{NiO}$ spins are fully rotatable. Due to strong interfacial exchange coupling to neighboring $\mathrm{Fe}$ magnetic moments, the orientation of AFM NiO spins can be switched by small external magnetic fields on the order of only $500 \mathrm{Oe}$, orders of magnitude below spin-flop fields reported for magnetically isolated antiferromagnets.

\section{ACKNOWLEDGMENTS}

This work was founded by the National Science Centre, Poland, under Grant No. 2020/38/E/ST3/00086. This work is part of the scientific activities of the CERIC-ERIC internal project MAG-ALCHEMI.
[1] S. A. Wolf, D. D. Awschalom, R. A. Buhrman, J. M. Daughton, S. Von Molnár, M. L. Roukes, A. Y. Chtchelkanova, and D. M. Treger, Spintronics: A spin-based electronics vision for the future, Science 294, 1488 (2001).

[2] V. Baltz, A. Manchon, M. Tsoi, T. Moriyama, T. Ono, and Y. Tserkovnyak, Antiferromagnetic spintronics, Rev. Mod. Phys. 90, 015005 (2018).

[3] B. G. Park, J. Wunderlich, X. Martí, V. Holý, Y. Kurosaki, M. Yamada, H. Yamamoto, A. Nishide, J. Hayakawa, H. Takahashi, A. B. Shick, and T. Jungwirth, A spin-valve-like magnetoresistance of an antiferromagnet-based tunnel junction, Nat. Mater. 10, 347 (2011).

[4] X. Marti, I. Fina, C. Frontera, J. Liu, P. Wadley, Q. He, R. J. Paull, J. D. Clarkson, J. Kudrnovský, I. Turek, J. Kuneš, D. Yi, J. H. Chu, C. T. Nelson, L. You, E. Arenholz, S. Salahuddin, J. Fontcuberta, T. Jungwirth, and R. Ramesh, Room-temperature antiferromagnetic memory resistor, Nat. Mater. 13, 367 (2014).

[5] S. Nakatsuji, N. Kiyohara, and T. Higo, Large anomalous Hall effect in a non-collinear antiferromagnet at room temperature, Nature (London) 527, 212 (2015).

[6] Y. Cheng, S. Yu, M. Zhu, J. Hwang, and F. Yang, Electrical Switching of Tristate Antiferromagnetic Néel Order in $\alpha-\mathrm{Fe}_{2} \mathrm{O}_{3}$ Epitaxial Films, Phys. Rev. Lett. 124, 027202 (2020).

[7] P. Wadley, B. Howells, J. Železný, C. Andrews, V. Hills, R. P. Campion, V. Novák, K. Olejník, F. Maccherozzi, S. S. Dhesi, S. Y. Martin, T. Wagner, J. Wunderlich, F. Freimuth, Y. Mokrousov, J. Kuneš, J. S. Chauhan, M. J. Grzybowski, A. W. Rushforth, K. Edmond, B. L. Gallagher, and T. Jungwirth, Spintronics: Electrical switching of an antiferromagnet, Science 351, 587 (2016).

[8] J. Železný, P. Wadley, K. Olejník, A. Hoffmann, and H. Ohno, Spin transport and spin torque in antiferromagnetic devices, Nat. Phys. 14, 220 (2018).

[9] H. Yan, Z. Feng, P. Qin, X. Zhou, H. Guo, and X. Wang, Electric-field-controlled antiferromagnetic spintronic devices, Adv. Mater. 32, 1905603 (2020).

[10] H. Yan, Z. Feng, S. Shang, X. Wang, Z. Hu, J. Wang, Z. Zhu, H. Wang, Z. Chen, H. Hua, W. Lu, J. Wang, P. Qin, H. Guo, X. Zhou, Z. Leng, Z. Liu, C. Jiang, M. Coey, and Z. Liu, A piezoelectric, strain-controlled antiferromagnetic memory insensitive to magnetic fields, Nat. Nanotechnol. 14, 131 (2019).

[11] J. Barker and O. A. Tretiakov, Static and Dynamical Properties of Antiferromagnetic Skyrmions in the Presence of Applied Current and Temperature, Phys. Rev. Lett. 116, 147203 (2016).
[12] X. Zhang, Y. Zhou, and M. Ezawa, Antiferromagnetic skyrmion: Stability, creation and manipulation, Sci. Rep. 6, 24795 (2016).

[13] S. M. Wu, W. Zhang, A. KC, P. Borisov, J. E. Pearson, J. S. Jiang, D. Lederman, A. Hoffmann, and A. Bhattacharya, Antiferromagnetic Spin Seebeck Effect, Phys. Rev. Lett. 116, 097204 (2016).

[14] C. Sürgers, T. Wolf, P. Adelmann, W. Kittler, G. Fischer, and H. V. Löhneysen, Switching of a large anomalous Hall effect between metamagnetic phases of a non-collinear antiferromagnet, Sci. Rep. 7, 42982 (2017).

[15] M. Misiorny, M. Hell, and M. R. Wegewijs, Spintronic magnetic anisotropy, Nat. Phys. 9, 801 (2013).

[16] M. S. Wörnle, P. Welter, M. Giraldo, T. Lottermoser, M. Fiebig, P. Gambardella, and C. L. Degen, Coexistence of Bloch and Néel walls in a collinear antiferromagnet, Phys. Rev. B 103, 094426 (2021).

[17] C. Song, Y. You, X. Chen, X. Zhou, Y. Wang, and F. Pan, How to manipulate magnetic states of antiferromagnets, Nanotechnology 29, 112001 (2018).

[18] J. Nogués, J. Sort, S. Suriñach, J. S. Muñoz, M. D. Baró, J. F. Bobo, U. Lüders, E. Haanappel, M. R. Fitzsimmons, A. Hoffmann, and J. W. Cai, Isothermal tuning of exchange bias using pulsed fields, Appl. Phys. Lett. 82, 3044 (2003).

[19] F. L. A. Machado, P. R. T. Ribeiro, J. Holanda, R. L. RodríguezSuárez, A. Azevedo, and S. M. Rezende, Spin-flop transition in the easy-plane antiferromagnet nickel oxide, Phys. Rev. B 95, 104418 (2017).

[20] W. Kuch, L. I. Chelaru, F. Offi, J. Wang, M. Kotsugi, and J. Kirschner, Tuning the magnetic coupling across ultrathin antiferromagnetic films by controlling atomic-scale roughness, Nat. Mater. 5, 128 (2006).

[21] A. Scholl, M. Liberati, E. Arenholz, H. Ohldag, and J. Stöhr, Creation of an Antiferromagnetic Exchange Spring, Phys. Rev. Lett. 92, 247201 (2004).

[22] A. Kozioł-Rachwał, M. Slęzak, M. Zajac, P. Drózdz, W. Janus, M. Szpytma, H. Nayyef, and T. Slęzak, Control of spin orientation in antiferromagnetic $\mathrm{NiO}$ by epitaxial strain and spin-flop coupling, APL Mater. 8, 061107 (2020).

[23] A. A. Sapozhnik, R. Abrudan, Y. Skourski, M. Jourdan, H. Zabel, M. Kläui, and H. J. Elmers, Manipulation of antiferromagnetic domain distribution in $\mathrm{Mn}_{2} \mathrm{Au}$ by ultrahigh magnetic fields and by strain, Phys. Status Solidi - Rapid Res. Lett. 11, 1600438 (2017). 
[24] A. A. Sapozhnik, M. Filianina, S. Y. Bodnar, A. Lamirand, M. A. Mawass, Y. Skourski, H. J. Elmers, H. Zabel, M. Kläui, and M. Jourdan, Direct imaging of antiferromagnetic domains in $\mathrm{Mn} 2 \mathrm{Au}$ manipulated by high magnetic fields, Phys. Rev. B 97, 134429 (2018).

[25] W. H. Meiklejohn and C. P. Bean, New magnetic anisotropy, Phys. Rev. 105, 904 (1957).

[26] J. Nogués and I. K. Schuller, Exchange bias, J. Magn. Magn. Mater. 192, 203 (1999).

[27] M. D. Stiles and R. D. McMichael, Coercivity in exchange-bias bilayers, Phys. Rev. B 63, 064405 (2001).

[28] M. Estrader, A. López-Ortega, I. V. Golosovsky, S. Estradé, A. G. Roca, G. Salazar-Alvarez, L. López-Conesa, D. Tobia, E. Winkler, J. D. Ardisson, W. A. A. Macedo, A. Morphis, M. Vasilakaki, K. N. Trohidou, A. Gukasov, I. Mirebeau, O. L. Makarova, R. D. Zysler, F. Peiró, M. D. Baró, L. Bergström, and J. Nogués, Origin of the large dispersion of magnetic properties in nanostructured oxides: $\mathrm{FexO} / \mathrm{Fe}_{3} \mathrm{O}_{4}$ nanoparticles as a case study, Nanoscale 7, 3002 (2015).

[29] H. Matsuyama, C. Haginoya, and K. Koike, Microscopic Imaging of Fe Magnetic Domains Exchange-Coupled with Those in a NiO(001) Surface, Phys. Rev. Lett. 85, 646 (2000).

[30] J. Wu, D. Carlton, J. S. Park, Y. Meng, E. Arenholz, A. Doran, A. T. Young, A. Scholl, C. Hwang, H. W. Zhao, J. Bokor, and Z. Q. Qiu, Direct observation of imprinted antiferromagnetic vortex states in $\mathrm{CoO} / \mathrm{Fe} / \mathrm{Ag}(001)$ discs, Nat. Phys. 7, 303 (2011).

[31] H. Ohldag, H. Shi, E. Arenholz, J. Stöhr, and D. Lederman, Parallel versus Antiparallel Interfacial Coupling in Exchange Biased Co/FeF2, Phys. Rev. Lett. 96, 027203 (2006).

[32] D. A. Gilbert, J. Olamit, R. K. Dumas, B. J. Kirby, A. J. Grutter, B. B. Maranville, E. Arenholz, J. A. Borchers, and K. Liu, Controllable positive exchange bias via redox-driven oxygen migration, Nat. Commun. 7, 11050 (2016).

[33] N. Koon, Calculations of Exchange Bias in Thin Films with Ferromagnetic/Antiferromagnetic Interfaces, Phys. Rev. Lett. 78, 4865 (1997).

[34] T. J. Moran, J. Nogués, D. Lederman, and I. K. Schuller, Perpendicular coupling at $\mathrm{Fe}-\mathrm{FeF}_{2}$ interfaces, Appl. Phys. Lett. 72, 617 (1998).

[35] J. Wu, J. S. Park, W. Kim, E. Arenholz, M. Liberati, A. Scholl, Y. Z. Wu, C. Hwang, and Z. Q. Qiu, Direct Measurement of Rotatable and Frozen CoO Spins in Exchange Bias System of $\mathrm{CoO} / \mathrm{Fe} / \mathrm{Ag}(001)$, Phys. Rev. Lett. 104, 217204 (2010).

[36] J. S. Park, J. Wu, E. Arenholz, M. Liberati, A. Scholl, Y. Meng, and C. Hwang, Rotatable magnetic anisotropy of $\mathrm{CoO} / \mathrm{Fe} / \mathrm{Ag}(001)$ in ultrathin regime of the $\mathrm{CoO}$ layer, Appl. Phys. Lett. 97, 042505 (2010).

[37] W. L. Roth, Magnetic structures of $\mathrm{MnO}, \mathrm{FeO}, \mathrm{CoO}$, and $\mathrm{NiO}$, Phys. Rev. 110, 1333 (1958).

[38] I. Gray, T. Moriyama, N. Sivadas, G. M. Stiehl, J. T. Heron, R. Need, B. J. Kirby, D. H. Low, K. C. Nowack, D. G. Schlom, D. C. Ralph, T. Ono, and G. D. Fuchs, Spin Seebeck Imaging of Spin-Torque Switching in Antiferromagnetic Pt/NiO Heterostructures, Phys. Rev. X 9, 041016 (2019).

[39] H. J. Elmers and U. Gradmann, Magnetic anisotropies in Fe(110) films on W(110), Appl. Phys. A 51, 255 (1990).

[40] U. Gradmann, J. Korecki, and G. Waller, In-plane magnetic surface anisotropies in Fe(110), Appl. Phys. A 39, 101 (1986).
[41] T. Ślęzak, M. Ślęzak, M. Zając, K. Freindl, A. Kozioł-Rachwał, K. Matlak, N. Spiridis, D. Wilgocka-Ślęzak, E. PartykaJankowska, M. Rennhofer, A. I. Chumakov, S. Stankov, R. Rüffer, and J. Korecki, Noncollinear Magnetization Structure at the Thickness-Driven Spin-Reorientation Transition in Epitaxial Fe Films on W(110), Phys. Rev. Lett. 105, 027206 (2010).

[42] L. Baldrati, O. Gomonay, A. Ross, M. Filianina, R. Lebrun, R. Ramos, C. Leveille, F. Fuhrmann, T. R. Forrest, F. MacCherozzi, S. Valencia, F. Kronast, E. Saitoh, J. Sinova, and M. Klaüi, Mechanism of Néel Order Switching in Antiferromagnetic Thin Films Revealed by Magnetotransport and Direct Imaging, Phys. Rev. Lett. 123, 177201 (2019).

[43] M. Dąbrowski, T. Nakano, D. M. Burn, A. Frisk, D. G. Newman, C. Klewe, Q. Li, M. Yang, P. Shafer, E. Arenholz, T. Hesjedal, G. van der Laan, Z. Q. Qiu, and R. J. Hicken, Coherent Transfer of Spin Angular Momentum by Evanescent Spin Waves within Antiferromagnetic NiO, Phys. Rev. Lett. 124, 217201 (2020).

[44] H. Qiu, L. Zhou, C. Zhang, J. Wu, Y. Tian, S. Cheng, S. Mi, H. Zhao, Q. Zhang, D. Wu, B. Jin, J. Chen, and P. Wu, Ultrafast spin current generated from an antiferromagnet, Nat. Phys. 17, 388 (2021).

[45] C. Jensen, K. Reshöft, and U. Köhler, Direct observation of strain relaxation in iron layers on W(1 10$)$ by time-resolved STM, Appl. Phys. A: Mater. Sci. Process. 62, 217 (1996).

[46] E. Vescovo, T. O. Menteş, J. T. Sadowski, J. M. Ablett, M. A. Niño, and A. Locatelli, Domain faceting in an in-plane magnetic reorientation transition, Phys. Rev. B 82, 184405 (2010).

[47] Y. Zhang, P. Buczek, L. Sandratskii, W. X. Tang, J. Prokop, I. Tudosa, T. R. F. Peixoto, K. Zakeri, and J. Kirschner, Nonmonotonic thickness dependence of spin wave energy in ultrathin $\mathrm{Fe}$ films: Experiment and theory, Phys. Rev. B: Condens. Matter Mater. Phys. 81, 094438 (2010).

[48] U. Gradmann, T. Dürkop, and H. J. Elmers, Magnetic moments and anisotropies in smooth and rough surfaces and interfaces, J. Magn. Magn. Mater. 165, 56 (1997).

[49] I.-G. Baek, H. G. Lee, H.-J. Kim, and E. Vescovo, Spin reorientation transition in $\mathrm{Fe}(110)$ thin films: The role of surface anisotropy, Phys. Rev. B 67, 075401 (2003).

[50] M. Ślęzak, P. Dróżdż, K. Matlak, A. Kozioł-Rachwał, J. Korecki, and T. Ślęzak, Multiple spin reorientation transitions and large in plane magnetic anisotropy in epitaxial $\mathrm{Au} / \mathrm{Co} / \mathrm{Fe}(110)$ films, J. Magn. Magn. Mater. 475, 195 (2019).

[51] M. Ślęzak, T. Ślęzak, K. Matlak, B. Matlak, P. Dróżdż, T. Giela, D. Wilgocka-Ślęzak, N. Pilet, J. Raabe, A. Kozioł-Rachwał, and J. Korecki, Giant in-plane magnetic anisotropy in epitaxial bcc $\mathrm{Co} / \mathrm{Fe}(110)$ bilayers, Phys. Rev. B 94, 014402 (2016).

[52] M. Ślęzak, T. Giela, D. Wilgocka-Ślęzak, A. Kozioł-Rachwał, T. Ślęzak, R. Zdyb, N. Spiridis, C. Quitmann, J. Raabe, N. Pilet, and J. Korecki, X-Ray photoemission electron microscopy study of the in-plane spin reorientation transitions in epitaxial Fe films on W(110), J. Magn. Magn. Mater. 348, 101 (2013).

[53] F. Gerhardter, Y. Li, and K. Baberschke, Temperaturedependent ferromagnetic-resonance study in ultrahigh vacuum: Magnetic anisotropies of thin iron films, Phys. Rev. B 47, 11204 (1993).

[54] M. Ślęzak, T. Ślęzak, P. Dróżdż, B. Matlak, K. Matlak, A. Kozioł-Rachwał, M. Zając, and J. Korecki, How a ferromagnet drives an antiferromagnet in exchange biased $\mathrm{CoO} / \mathrm{Fe}(110)$ bilayers, Sci. Rep. 9, 889 (2019). 
[55] M. Ślęzak, P. Dróżdż, A. Kozioł-Rachwał, K. Matlak, J. Korecki, M. Zając, and T. Ślęzak, Magnetic anisotropy and temperature dependence of exchange bias in epitaxial $\mathrm{CoO}(111) / \mathrm{Fe}(110)$ bilayers, Acta Phys. Pol. A 137, 44 (2020).

[56] M. Ślęzak, P. Drózdz, W. Janus, H. Nayyef, A. Kozioł-Rachwał, M. Szpytma, M. Zając, T. O. Menteş, F. Genuzio, A. Locatelli, and T. Ślęzak, Fine tuning of ferromagnet/antiferromagnet interface magnetic anisotropy for field-free switching of antiferromagnetic spins, Nanoscale 12, 18091 (2020).

[57] M. Zajacc, T. Giela, K. Freindl, K. Kollbek, J. Korecki, E. Madej, K. Pitala, A. Kozioł-Rachwał, M. Sikora, N. Spiridis, J. Stępień, A. Szkudlarek, M. Ślęzak, T. Ślęzak, and D. Wilgocka-Ślęzak, The first experimental results from the 04BM (PEEM/XAS) beamline at Solaris, Nucl. Instrum. Methods Phys. Res., Sect. B 492, 43 (2021).

[58] T. O. Menteş, G. Zamborlini, A. Sala, and A. Locatelli, Cathode lens spectromicroscopy: Methodology and applications, Beilstein J. Nanotechnol. 5, 1873 (2014).

[59] See Supplemental Material at http://link.aps.org/supplemental/ 10.1103/PhysRevB.104.134434 for additional LEED, XMCD and XMLD results.

[60] E. Arenholz, G. Van Der Laan, R. V. Chopdekar, and Y. Suzuki, Angle-Dependent Ni 2+ X-Ray Magnetic Linear Dichroism: Interfacial Coupling Revisited, Phys. Rev. Lett. 98, 197201 (2007).
[61] Y. Z. Wu, Y. Zhao, E. Arenholz, A. T. Young, B. Sinkovic, C. Won, and Z. Q. Qiu, Analysis of x-ray linear dichroism spectra for $\mathrm{NiO}$ thin films grown on vicinal $\mathrm{Ag}(001)$, Phys. Rev. B: Condens. Matter Mater. Phys. 78, 064413 (2008).

[62] E. Arenholz, G. Van Der Laan, and F. Nolting, Magnetic structure near the CoNiO (001) interface, Appl. Phys. Lett. 93, 162506 (2008).

[63] W. Zhu, L. Seve, R. Sears, B. Sinkovic, and S. S. P. Parkin, Field Cooling Induced Changes in the Antiferromagnetic Structure of NiO Films, Phys. Rev. Lett. 86, 5389 (2001).

[64] D. Alders, L. H. Tjeng, F. C. Voogt, T. Hibma, G. A. Sawatzky, C. T. Chen, J. Vogel, M. Sacchi, and S. Iacobucci, Temperature and thickness dependence of magnetic moments in NiO epitaxial films, Phys. Rev. B: Condens. Matter Mater. Phys. 57, 11623 (1998).

[65] S. Saito, M. Miura, K. Kurosawa, H. Van Der Heide, and C. F. Van Bruggen, Magnetic torque measurements on $\mathrm{NiO}(111)$ platelets, J. Phys. C: Solid State Phys. 13, 1521 (1980).

[66] J. Geshev, L. Pereira, and J. Schmidt, Rotatable anisotropy and coercivity in exchange-bias bilayers, Phys. Rev. B 66, 134432 (2002).

[67] O. Fruchart, J. P. Nozieres, and D. Givord, Temperature-driven in-plane anisotropy reorientation transition in $\mathrm{Fe}(110)$ films, J. Magn. Magn. Mater. 165, 508 (1997). 